\title{
Trivium
}

Revue franco-allemande de sciences humaines et sociales - Deutsch-französische Zeitschrift für Geistesund Sozialwissenschaften

$33 \mid 2021$

Concepts historiques fondamentaux - Démocratie

\section{Die Geschichtlichen Grundbegriffe. Allgemeine Einführung}

\section{Catherine Colliot-Thélène et Élisabeth Kauffmann}

Traducteur : Bernd Schwibs

\section{CpenEdition}

Journals

Édition électronique

URL : https://journals.openedition.org/trivium/7729

DOI : $10.4000 /$ trivium. 7729

ISSN : 1963-1820

Éditeur

Les éditions de la Maison des sciences de l'Homme

Référence électronique

Catherine Colliot-Thélène und Élisabeth Kauffmann, „Die Geschichtlichen Grundbegriffe. Allgemeine Einführung“, Trivium [Online], 33 | 2021, online erschienen am 07 Juni 2021, abgerufen am 10 Juni 2021. URL: http://journals.openedition.org/trivium/7729 ; DOI: https://doi.org/10.4000/trivium.7729

Ce document a été généré automatiquement le 10 juin 2021.

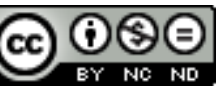

Les contenus des la revue Trivium sont mis à disposition selon les termes de la Licence Creative Commons Attribution - Pas d'Utilisation Commerciale - Pas de Modification 4.0 International. 


\title{
Die Geschichtlichen Grundbegriffe. Allgemeine Einführung
}

\author{
Catherine Colliot-Thélène et Élisabeth Kauffmann \\ Traduction : Bernd Schwibs
}

Die Geschichtlichen Grundbegriffe, herausgegeben von Otto Brunner (1898-1986), Werner Conze (1910-1986) und Reinhart Koselleck (1923-2006), umfassen neun zwischen 1972 und 1997 erschienene Bände. ${ }^{1}$ Der Untertitel, Historisches Lexikon zur politisch-sozialen Sprache in Deutschland, weist das Unternehmen zugleich als Lexikon und als Enzyklopädie aus: als Lexikon der alphabetisch geordneten Wörter, in denen Politik und Gesellschaft sich von der Antike bis ins 20. Jahrhundert geäußert haben; und als Enzyklopädie der Vor- und Darstellungen, Verhaltensweisen, sozialen und politischen Strukturen sowie der diesen Wörtern entsprechenden Kenntnisse. Der Leitfaden der Analysen, die Wörter, ihre semantischen Veränderungen und Verschiebungen im Lauf der Jahrhunderte, impliziert, dass diese Arbeit sich zwangsläufig an einem spezifischen Sprachkorpus vollzieht: der politischen und sozialen Sprache in Deutschland. Dabei wird selbstredend auf Altgriechisch und Latein Bezug genommen, da eine Vielzahl der Wörter bzw. Begriffe in der einen oder anderen dieser beiden Sprachen wurzelt. Aufgrund der Verflochtenheit der sozialen und politischen Geschichte der Nationen Europas und der Zirkulation der Ideen und Vorstellungen zwischen ihnen wird zugleich auch auf Texte in den modernen Fremdsprachen, insbesondere dem Französischen, zurückgegriffen. Gleichwohl bleibt der Fokussierungspunkt aller Artikel die deutsche Terminologie und die Art und Weise, in der die Begriffe darin ihren Ausdruck und ihre Transformation erfahren.

Die Geschichtlichen Grundbegriffe bilden seit Jahrzehnten eine Fundgrube an Sachwissen, aus der nicht nur die deutschen Forscher geschöpft haben, sondern in Frankreich neben den Germanisten all jene, Historiker, Philosophen, Politikwissenschaftler und Soziologen, die der deutschen Sprache mächtig sind. Es ist wünschenswert, diesen Wissenskorpus einem breiteren Publikum zu öffnen. Angesichts des Umfangs des Gesamtwerks ist dessen vollständige Übersetzung allerdings schwer zu realisieren. Wir legen daher in einer Serie von Trivium-Nummern eine Auswahl von Begriffen vor, ${ }^{2}$ 
deren Kohärenz sich aus ihrer Lektüre im Verbund mit der je spezifischen Einleitung zu jedem von ihnen ergeben sollte. Die vorliegende allgemeine Einführung möchte lediglich die Originalität des Unternehmens dieser Gesellschaftlichen Grundbegriffe erhellen und unterstreichen: Im Gegensatz $\mathrm{zu}$ einer Ideen- oder einer Problemgeschichte, zwei Gattungen, die eine gewisse zeitliche Beständigkeit ihrer Gegenstände voraussetzen, zielt die Begriffsgeschichte darauf ab, anhand einer historischen Semantik die Wandlungsprozesse der sozialen und politischen Strukturen sowie der ihnen korrespondierenden Dar- und Vorstellungen sichtbar zu machen.

Über die entscheidende Rolle Reinhart Kosellecks bei der Konzeption des Projekts sollten die beiden anderen Herausgeber nicht vergessen werden, die vor Beendigung des Unternehmens verstarben. Der österreichische Historiker Otto Brunner, bekannt für seine Studien zum Spätmittelalter ${ }^{3}$ und Verfasser des Stichworts »Feudalismus« in den GG, hat Pionierarbeit geleistet im Hinblick auf die methodologischen Überlegungen zu einer Sozial- und Begriffsgeschichte. Mit dem Arbeitskreis für moderne Sozialgeschichte, ${ }^{4}$ den er 1957 in Heidelberg gründete und der bis heute besteht, war Werner Conze Initiator einer neuen Sozialgeschichte. Reinhart Koselleck war von 1960 bis 1965 wissenschaftlicher Mitarbeiter dieses Kreises, bevor er 1965 zum Mitglied gewählt und 1986 dessen Vorsitzender wurde. Unbestritten tragen die GG den Stempel dieser Sozialgeschichte, die Werner Conze nicht als Spartendisziplin konzipiert hatte, sondern als synthetische Herangehensweise an die geschichtliche Welt in ihrer Gesamtheit. ${ }^{5}$

4 Die Vaterschaft der Grundthese, auf der die GG aufgebaut sind, gebührt dennoch R. Koselleck. Dieser, der als einer der größten deutschen Historiker der zweiten Hälfte des 20. Jahrhunderts gilt, ist dank der Übersetzung mehrerer seiner Arbeiten auch in Frankreich bekannt: 1979 erschien die französische Übersetzung von Kritik und Krise, ${ }^{6}$ seine 1959 erschienene Doktorarbeit; 1990 die von Vergangene Zukunft. Zur Semantik geschichtlicher Zeiten; ${ }^{7}$ 1997, unter dem Titel L'Expérience de l'Histoire, ${ }^{8}$ ein Sammelband mit Texten zur Geschichtstheorie, darunter den Abschnitten V und VII des Stichworts "Geschichte« aus den GG, die das Gesamtunternehmen aufschlüsseln. Dem wäre noch ein in Trivium erschienener Artikel hinzuzufügen: „Gibt es eine Beschleunigung der Geschichte? «"

»Wozu noch Historie? ${ }^{10}{ }^{10}$ so fragte sich Koselleck in einem 1970 gehaltenen Vortrag, zwei Jahre vor der Veröffentlichung des ersten Bandes der GG. Diese Frage nach der Legitimität der Geschichtswissenschaft, weitergehend nach dem Begriff von Geschichte überhaupt, schwingt in allen seinen Arbeiten bis in die letzten Lebensjahre mit. Kritik und Krise widmete bereits ein längeres Kapitel der »bürgerlichen« Geschichtsphilosophie, der Koselleck zuschrieb, der Verschleierung der aufrührerischen Implikationen des moralischen Universalismus der Aufklärung Vorschub geleistet zu haben. Zwischen diesem ersten Werk und der Inangriffnahme der GG hat sich die Perspektive verändert. Kosellecks Bemühen gilt nun dem Aufweis eines Umschlagsprozesses im Geschichtsverständnis in der zweiten Hälfte des 18. Jahrhunderts und dessen Auswirkungen auf die politischen Begriffe. Dieser Umschlagsprozess ist mehr als eine bloße Episode in der Geschichte des Okzidents, mehr oder minder vergleichbar mit anderen. In Wahrheit handelt es sich um die Genese der Moderne, wie es Koselleck klar im Einleitungstext zum Gesamtwerk formuliert: „Die leitende Fragestellung ist, die Auflösung der alten und die Entstehung der modernen Welt in ihrer begrifflichen Erfassung zu untersuchen. ${ }^{11}$ 
6 An diesem Unternehmen war eine Vielzahl von Mitarbeitern beteiligt, mehrheitlich Historiker, aber auch Philosophen, Juristen, Theologen und Vertreter der Sozial- und Wirtschaftswissenschaften. Häufig $\mathrm{zu}$ mehreren verfasst, so die spezifischen Kompetenzen jedes Einzelnen verknüpfend, ist jedes Stichwort Ergebnis einer wirklichen Zusammenarbeit. Die Quellen, deutsche, aber auch griechische und lateinische, französische, englische und italienische, aus denen die Neuschöpfungen, Variationen, Äquivalente und Wandlungen der Begriffe gewonnen wurden, sind höchst unterschiedlich: Texte aus den Bereichen Jura, Philosophie, Theologie, Naturwissenschaft, Literatur und bisweilen Wirtschaft, Lexika und Enzyklopädien, desgleichen Pamphlete, Spottschriften und Zeitungsartikel. Die Homogenität der Fragestellung gewährleistet eine gemeinsame, chronologische Struktur, deren Schwerpunkt stets die Periode zwischen Anfang des 18. und Ende des 19. Jahrhunderts bildet. Denn die Hypothese lautet, in den Worten R. Kosellecks, »daß sich seit der Mitte des achtzehnten Jahrhunderts ein tiefgreifender Bedeutungswandel klassischer topoi vollzogen, daß alte Worte neue Sinngehalte gewonnen haben ${ }^{12}{ }^{12}$ sodass diese Epoche als eine Sattelzeit angesehen werden kann, in deren Verlauf Begriffe, die früher auf soziale und politische Bedingungen verwiesen, die seither umgewälzt wurden oder am Verschwinden begriffen sind, Bedeutungen gewannen, die uns heute vertraut sind. Die Auswahl der behandelten Begriffe gehorcht dem grundlegenden Interesse der Herausgeber des Gesamtwerks an dieser Sattelzeit (1750-1850), die anhand der in ihr auftretenden revolutionären Aufwallungen und sozialen Konflikte dem Begriffsrepertoire der modernen zeitgenössischen Epoche ihre Stempel aufprägte. Um Grundbegriffe handelt es sich insoweit, als sich in ihnen der gemeinsame Erfahrungsfundus kristallisiert, der in einer bestimmten Epoche (im vorliegenden Fall der neuzeitlichen) das wechselseitige Verständnis ermöglicht, das heißt ebenso Einverständnis wie Ausdruck der Divergenzen und Konflikte.

7 Der Kern der Umwälzung der Vorstellungen, die in dieser "Sattelzeit« die meisten der auf Gesellschaft und Politik bezogenen Begriffe tangiert, beruht in einer radikal neuen Anschauung von Historizität. Es versteht sich gewissermaßen von selbst, dass der Teil des Stichworts "Geschichte«, der die moderne Konzeption der Geschichte expliziert, von R. Koselleck verfasst wurde. Dieser zeigt, wie das Kollektivsingular "Geschichte« in Erscheinung trat und der Geschichtlichkeit Prozesscharakter verlieh. Die Geschichtsphilosophien des 18. und 19. Jahrhunderts erwiesen sich als gelehrter Ausdruck einer noch nicht dagewesenen Erfahrung von Zeit, die Koselleck zufolge aus einem wachsenden Auseinandertreten von "Erfahrungsraum» und "Erwartungshorizont" erwächst. Das Auseinanderklaffen von vergangenen Erfahrungen und Zukunftserwartungen ist Thema eines der Hauptartikel von Vergangene Zukunft. ${ }^{13}$ Eine seiner Auswirkungen ist insbesondere die "Temporalisierung" der Begriffe des sozialen und politischen Denkens, die sich teils durch das Auftreten neuer Begriffe (»Fortschritt« im allgemeinen, ohne Spezifizierung, "Entwicklung", "Zivilisation«), teils und häufiger durch den Wandel des Sinns überkommener Begriffe bekundet, die eine dynamische Dimension gewannen, welche sie in den allgemeinen Prozess einer zur Zukunft hin offenen Geschichte einschreibt. Die hier ausgewählten Artikel bieten einige Beispiele dafür. Um diesen allgemeinen Aspekt der die Analysen des Gesamtwerks der GG klar erkennbar zu machen, wurde besonders auf die Übersetzung der Schlüsselbegriffe geachtet, so die systematische Verwendung von "attente« für "Erwartung» oder das buchstäbliche, wenn auch im Französischen etwas ungelenke »concept de mouvement« für »Bewegungsbegriff«. 
Weitergehende Informationen hinsichtlich der Konzeption der Geschichtlichen Grundbegriffe, der Biographie Reinhart Kosellecks, der Kritiken und Kontroversen, die die Fragestellung der $G G$ ausgelöst hat, sowie $\mathrm{zu}$ weiterführenden Arbeiten französischer Forscher findet der Leser in den Arbeiten von Alexandre Escudier, ${ }^{14}$ François Hartog, ${ }^{15}$ Paul Ricœur ${ }^{16}$, im Sammelwerk Historicités ${ }^{17}$ sowie in der Nummer 25 der Revue germanique internationale, ${ }^{18}$ die zur Gänze Reinhart Koselleck gewidmet ist.

\section{BIBLIOGRAPHIE}

Barash, J. A. / Jollivet, S. (2017): Revue germanique internationale, Nr. 25: Reinhart Koselleck.

Brunner, O. / Conze, W. / Koselleck, R. (Hg.) (1972-1997): Geschichtliche Grundbegriffe: Historisches

Lexikon zur politisch-sozialen Sprache in Deutschland, Stuttgart: Klett-Cotta.

Brunner, O. (1965 [1939]): Land und Herrschaft: Grundfragen der territorialen Verfassungsgeschichte Südostdeutschlands im Mittelalter, 5. Aufl., Wien: Rohr.

Delacroix, Chr. / Dosse, F. / Garcia, P. (2009): Historicités, Paris: La Découverte.

Escudier, A. (2017): »)Temporalisation« et modernité politique: penser avec Koselleck«, Revue germanique internationale, 25, S. 37-67.

Escudier, A. (2020): »La \Sattelzeit`: genèse et contours d'un concept d'époque«, Éthique, politique, religions, 17 (2), S. 115-136.

Hartog, F. (2003): Régimes d'historicité. Présentisme et expériences du temps, Paris: Seuil.

Jollivet, S. (2017): »D'une radicalisation de l'historisme chez Reinhart Koselleck. Le projet renouvelé d'une théorie de l'histoire«, Revue germanique internationale, 25, S. 9-36.

Kocka, J. (1986): „Werner Conze und die Sozialgeschichte in der Bundesrepublik Deutschland «, Geschichte in Wissenschaft und Unterricht, 36, S. 595-602.

Koselleck, R. (1979): Vergangene Zukunft. Zur Semantik geschichtlicher Zeiten, Frankfurt/M.: Suhrkamp.

Koselleck, R. (1979 [1976]): »Erfahrungsraum Kategorien«, in: ders.: Vergangene Zukunft. Zur Semantik geschichtlicher Zeiten, S. 349-357.

Ricœur, P. (1991 [1985]): Zeit und Erzählung. Band III: Die erzählte Zeit, übers. von A. Knop, München: Fink.

\section{NOTES}

1. Brunner / Conze / Koselleck (1972-1997) (in der Folge GG). 2004 erschien eine preisgünstigere Studienausgabe.

2. Die Serie beginnt mit der Übersetzung von »Demokratie«; »Herrschaft«, »Macht/Gewalt« und "Staat/Souveränität» werden folgen. Der Artikel »Repräsentation« erschien in Übersetzung bereits in Trivum 16, 2014: https://journals.openedition.org/trivium/4781. 
3. Seine Studien zum Mittelalter wurzeln in der Landesgeschichte. Sein erstmals 1939 erschienenes Buch Land und Herrschaft: Grundfragen der territorialen Verfassungsgeschichte Südostdeutschlands im Mittelalter hat die deutsche Geschichtsschreibung durch sein Postulat quellennaher Konzeptualisierung nachhaltig beeinflusst.

4. http://www.ak-moderne-sozialgeschichte.de/.

5. Vgl. Kocka (1986).

6. Frz.: Le Règne de la critique. Étude sur la pathogenèse du monde bourgeois, übers. von H. Hildebrand, Paris: Éditions de Minuit, 1979.

7. Frz.: Le Futur passé: contribution à la sémantique des temps historiques, übers. von J. Hoock et M.Cl. Hoock, Paris : Éd. de l'EHESS, 1990, Neuauflage 2020.

8. L'Expérience de l'histoire, hg. und mit Vorwort von M. Werner, übers. von A. Escudier, in Zusammenarbeit mit D. Meur, M.-Cl. Hoock und J. Hoock, Paris: Gallimard, 1997 (Points, 2011).

9. »Y a-t-il une accélération du temps?«, Trivium, 9, 2011. Weitere Texte Kosellecks auf Französisch sind in diversen Gemeinschaftsveröffentlichungen verfügbar, darunter : ein Artikel »La désagrégation de la `maison` comme entité de domination« (dt. : »Die Auflösung des Hauses als ständischer Herrschaftseinheit «, Begriffsgeschichten), der 2009 in dem Sammelwerk Historicités erschien (hg. von C. Delacroix, F. Dosse und P. Garcia, Paris: La Découverte), ein Beitrag zu L'âge des révolutions européennes (1780-1848) (hg. von L. Bergeron, F. Furet, R. Koselleck, Paris: Bordas, 1973), ein Nachwort zu Charlotte Beradt, Rêver sous le III ${ }^{e}$ Reich, Paris : Payot et Rivages, 2002 (dt. Das Dritte Reich des Traums, München: Nymphenburger, 1966).

10. Erstmals 1971 erschienen in Historische Zeitschrift, Bd. 212, S. 1-18; aufgenommen in: ders., Vom Sinn und Unsinn der Geschichte, Berlin: Suhrkamp, 2010; S. 32-51. In dieser Frage klingt noch jener berühmte Vers Hölderlins aus »Brot und Wein« nach, »... und wozu Dichter in dürftiger Zeit«, den Heidegger in seinem Text »Wozu Dichter?« (Holzwege, Frankfurt/M.: Klostermann, 1950, S. 248-295) kommentiert hat.

11. Brunner / Conze / Koselleck (2004 [1972-1997]), Bd. 1, S. XIV, Anm. 1.

12. Brunner / Conze / Koselleck (2004 [1972-1997]), Bd. 1, S. XV, Anm. 1

13. Koselleck (1979 [1976]). Paul Ricœur hat sich im 3. Band von Zeit und Erzählung (1991, S. 33 ff.) auf diese Analysen gestützt.

14. Escudier (2017); (2020).

15. Hartog (2003).

16. Ricœur (1991 [1985]).

17. Delacroix / Dosse / Garcia (2009).

18. Barash / Jollivet (2017). Für eine Gesamtanalyse des Theorieprojekts von Koselleck, jenseits der GG, siehe insbesondere Escudier (2017) und Jollivet (2017).

\section{INDEX}

Mots-clés : Geschichtliche Grundbegriffe, Koselleck

Schlüsselwörter : Geschichtliche Grundbegriffe, Koselleck 


\section{AUTEURS}

\section{CATHERINE COLLIOT-THÉLÈNE}

Catherine Colliot-Thélène ist emeritierte Professorin für Philosophie an der Universität Rennes I. Nähere Informationen finden Sie hier.

\section{ÉLISABETH KAUFFMANN}

Élisabeth Kauffmann ist Übersetzerin und Herausgeberin philosophischer, epistemologischer und soziologischer Texte. Nähere Informationen finden Sie hier. 